\title{
Effects of total solar eclipse of 29 March 2006 on surface radiation
}

\author{
S. Kazadzis ${ }^{1}$, A. Bais ${ }^{1}$, M. Blumthaler ${ }^{2}$, A. Webb ${ }^{3}$, N. Kouremeti ${ }^{1}$, R. Kift ${ }^{3}$, B. Schallhart ${ }^{2}$, and A. Kazantzidis ${ }^{1}$ \\ ${ }^{1}$ Laboratory of Atmospheric Physics, Aristotle University of Thessaloniki, Greece \\ ${ }^{2}$ Division of Biomedical Physics, Innsbruck Medical University, Austria \\ ${ }^{3}$ School of Earth Atmospheric and Environmental Sciences, University of Manchester, UK
}

Received: 1 June 2007 - Published in Atmos. Chem. Phys. Discuss.: 29 June 2007

Revised: 24 October 2007 - Accepted: 1 November 2007 - Published: 22 November 2007

\begin{abstract}
Solar irradiance spectral measurements were performed during a total solar eclipse. The spectral effect of the limb darkening to the global, direct irradiance and actinic flux measurements was investigated. This effect leads to wavelength dependent changes in the measured solar spectra showing a much more pronounced decrease in the radiation at the lower wavelengths. Radiative transfer model results were used for the computation of a correction for the total ozone measurements due to the limb darkening. This correction was found too small to explain the large decrease in total ozone column derived from the standard Brewer measurements, which is an artifact in the measured irradiance due to the increasing contribution of diffuse radiation against the decreasing direct irradiance caused by the eclipse. Calculations of the Extraterrestrial spectrum and the effective sun's temperatures, as measured from ground based direct irradiance measurements, showed an artificial change in the calculations of both quantities due to the fact that radiation coming from the visible part of the sun during the eclipse phases differs from the black body radiation described by the Planck's law.
\end{abstract}

\section{Introduction}

On 29 March 2006 a total solar eclipse was visible along a narrow corridor which traversed half the Earth, starting in Brazil, extending across the Atlantic, through North Africa, and central Asia and ending in northern Mongolia. The umbra traversed the Mediterranean passing directly over the Greek island of Kastelorizo $\left(36.150^{\circ} \mathrm{N}, 29.596^{\circ} \mathrm{E}\right)$.

The spectral behaviour of solar radiation reaching the earth's surface during the course of a solar eclipse can be studied either with ground based (GB) measurements or with

Correspondence to: S. Kazadzis

(skaza@skiathos.physics.auth.gr) the use of radiative transfer model (RTM) calculations to measure or simulate different radiation quantities. During an eclipse, as the disk of the moon moves across the sun its coverage of the limb and centre of the sun varies. Thus, "limb darkening" (LD) becomes relevant and needs to be taken into account. Measurements of radiative quantities (Beletsky et al., 1998; Jerlov et al., 1954; Sharp et al., 1971) and model calculations (Köpke et al., 2001) have been performed during various eclipse events.

There are only a few studies (Fernandez et al., 1993; Mikhalev et al., 1999) that present the eclipse-induced changes in the spectral solar UV irradiance at the earth's surface, and even fewer measurements of the extraterrestrial (ET) spectrum at UV wavelengths, exist (Show et al., 1978; Greve and Neckel, 1996; Köpke et al., 2001; Emde and Mayer, 2007).

Several earlier studies have examined possible effects of a solar eclipse on the ozone column (Bojkov, 1968; Chakrabarty et al., 1997; Jerlov et al., 1954; Svensson, 1958; Zerefos et al., 2001). Ozone observations with Brewer and Dobson instruments have shown a decrease in ozone during the maximum phase of the eclipse. Other studies, however, in which other instruments were used for the determination of total ozone, have shown different results as to the sign and the magnitude of total ozone changes. Bojkov (1968) reported results from Dobson spectrophotometric observations during the solar eclipse of May 1966 and concluded that an increase of 14 Dobson units (DU) was observed at the maximum phase of the eclipse. In that paper a review of total ozone measurements during a solar eclipse was presented, and it was emphasized that similar results were also reported in other studies based on Dobson ozone observations (Svensson, 1958; Stranz, 1961).

In this study we investigate the effect of the solar eclipse on measurements of several radiation quantities during a two-day campaign at Kastelorizo. Such quantities are: global irradiance (GI), which is the irradiance measured on

Published by Copernicus Publications on behalf of the European Geosciences Union. 
Table 1. Data of the eclipse timing at Kastelorizo.

\begin{tabular}{ll}
\hline Status of the eclipse & Time (UT) \\
\hline Beginning of eclipse & $09: 34: 46$ \\
Beginning of totality & $10: 51: 56$ \\
Maximum & $10: 53: 28$ \\
End of totality & $10: 55: 00$ \\
End of eclipse & $12: 10: 47$ \\
\hline
\end{tabular}

a horizontal surface, the direct irradiance (DI), which represents the direct sun irradiance component and actinic flux (AF), which represents the radiation measured by a spherical surface. Also, total column ozone was derived using the DI spectral measurements at selected wavelengths. In addition, RTM System for Transfer of Atmospheric Radiation (STAR) calculations of the ET solar spectrum proposed by Köpke et al. (2001) were used in order to investigate temporal and spectral variability of UV irradiance during the solar eclipse of 29 March 2006.

\section{Campaign information, instruments and modeling tools}

\subsection{Site description and eclipse details}

The campaign took place at the island of Kastelorizo, Greece, during 28 and 29 (eclipse day) March 2006, with the participation of three groups: Innsbruck Medical University, Division of Biomedical Physics, Austria (UI), Aristotle University, Physics Department, Thessaloniki, Greece (AUTH) and School of Earth Atmospheric and Environmental Sciences, University of Manchester, UK (UMAN). The instruments were deployed in a field on the north side of the island. Hills obstructed the horizon towards the south and east up to an elevation of about $20^{\circ}$, and affected the measurements of GI but caused no obstruction to viewing the sun during the eclipse, as well as for several hours before and after.

The timing of the different phases of the eclipse for the specific location is given in Table 1. In all figures, vertical lines mark the beginning, maximum (totality) and the end of the eclipse.

The weather on 28 March was clear with cloudless skies and excellent visibility. On 29 March cirrus clouds started to form after the beginning of the eclipse. Occasionally, the cirrus clouds obscured the sun, especially after the time of the totality, affecting mainly the measurements of the DI. A few minutes before totality, cumulus clouds started developing, and continued growing as the eclipse progressed. However, on several occasions it was still possible to see the sun and measure the DI.

\subsection{Instrumentation}

Various instruments were deployed at Kastelorizo during this two-day campaign. A double monochromator spectroradiometer (Bentham DTM 300-UI), operating in the spectral range $290-400 \mathrm{~nm}$ in steps of $0.5 \mathrm{~nm}$, was used to measure simultaneously GI, AF and in addition DI. The full width at half maximum (FWHM) for the GI and AF measurements was $0.95 \mathrm{~nm}$, while for the DI it was $0.55 \mathrm{~nm}$. The scanning time for a full spectrum was about $3 \mathrm{~min}$. A second double monochromator spectroradiometer (Brewer MK III-AUTH) was used to measure GI and DI in the wavelength region of $290-366 \mathrm{~nm}$ in steps of $0.5 \mathrm{~nm}$ and with spectral resolution of $0.55 \mathrm{~nm}(\mathrm{FWHM})$. For this study the operating software of the instrument was modified to allow the alternating measurement (every $30 \mathrm{~s}$ ) of global and direct spectral irradiance at 6 wavelengths between 302 and $320 \mathrm{~nm}$. The two instruments have been used in many UV monitoring campaigns measuring GI, DI and AF (Bais et al., 2001; Kylling et al., 2005; Webb et al., 2002). From the DI measurements the total ozone column was calculated using the standard Brewer algorithm (differential absorption method). The absolute calibration of both instruments is based on lamps of spectral irradiance traceable to the Physikalisch-Technische Bundesanstalt standards. However, in this campaign the emphasis was given to the relative change of the radiation due to the eclipse, and therefore the absolute calibration of the measurements was less important.

Two diode arrays (PDA-UMAN) and a coupled charged device (CCD-AUTH) spectrometers (all single monochromators) were also used to record spectral measurements of GI, DI, AF and zenith radiance. Their advantage is the instantaneous recording of the spectrum, which eliminates distortions from clouds or from the change in the ET irradiance during the eclipse. The CCD was used for measurements of the DI and the sky radiance in the spectral range of 280-1040 nm with a variable wavelength dependent FWHM (ranging between $1.9 \mathrm{~nm}$ and $3.5 \mathrm{~nm}$ ). Its usual sampling rate is one measurement every $2 \mathrm{~s}$ and the field of view is $1.2^{\circ}$. The instrument was mounted on a tracking system capable of following the sun. The first PDA instrument (Ocean Optics S2000) was used for measurements of the GI in the spectral range of 290-850 nm with a FWHM of $1.3 \mathrm{~nm}$, measuring one spectrum every $12 \mathrm{~s}$. A second channel measured zenith sky radiance. The second PDA (Metcon Inc.) was used for measurements of the actinic flux in the spectral range of 290$700 \mathrm{~nm}$ with a FWHM of $2.3 \mathrm{~nm}$, measuring one spectrum every $8 \mathrm{~s}$. A second channel measured DI in parallel with the Bentham.

In addition to the spectral measurements, measurements with broadband radiometers were also available. A NILU (Norsk Intitutt for Luftforskning) ultraviolet multi-filter radiometer (owned by AUTH) measured GI at 5 narrow bands $(\sim 10 \mathrm{~nm}$ FWHM) in the UVB $(305,312$, and $320 \mathrm{~nm})$, the UVA (340 and $380 \mathrm{~nm}$ ), and the photosynthetically active 
radiation (PAR, 400-700 nm) with a frequency of $1 \mathrm{~Hz}$. In addition, an erythemal detector, a UVA detector and a pyranometer operated continuously, recording $1 \mathrm{~min}$ averages.

Direct sun measurements with 2 handheld sunphotometers (Microtops) with filters centred at 300, $305,312,340,380,440,500,675,940$ and $1020 \mathrm{~nm}$ were used to derive total ozone and aerosol optical depth. Manual measurements were made about every half hour during the day increasing in frequency to every few minutes during the eclipse. Two cameras with fish-eye lenses were taking pictures of the full sky; one camera was equipped with an additional adjustable polarization filter. Pictures were taken every $10 \mathrm{~min}$, with an increased frequency of one picture per min during the eclipse.

The uncertainty on the absolute level of the quantities of all instruments described above is less than $10 \%$, while for the spectroradiometric measurements is less than $5 \%$. Since most of the issues discussed in this paper are described using ratios of quantities measured by the same instrument or relative changes of GI, DI or AF, the uncertainty on the results shown is expected to be lower than the above percentages.

2.3 Modelling of the extraterrestrial irradiance spectrum during the eclipse

To determine solar radiation quantities reaching the Earth's surface, the ET solar radiation is used, for which the scattering and absorption processes can be spectrally modeled. In general the ET solar irradiance at each wavelength is given as an average over the whole solar disc. During different eclipse periods, the moon covers different parts of the limb and the center of the sun. Thus the spectral dependence of the limb darkening of the sun becomes relevant. For the conditions of the total eclipse in Kastelorizo, this change of ET solar irradiance was calculated with help of one dimensional (1-D) radiative transfer model calculations presented in Köpke et al. (2001). The model calculations have been carried out for both eclipse and non-eclipse conditions to obtain the variation of various radiative quantities during the eclipse, under real atmospheric conditions but without the presence of clouds. The simulations of the radiative processes in the atmosphere have been performed by an improved version of the radiative transfer model STAR (Schwander et al., 2001). It is based on a one-dimensional radiative transfer algorithm (Nakajima and Tanaka, 1988) that considers absorption and scattering (including multiple scattering) of air molecules, aerosol particles and atmospheric trace gases. The formulas used for taking into account the LD effect, taken from Waldmeier (1941) and Scheffler and Elsässer (1974), are based on the geometric considerations of the position of the moon relative to the sun during the eclipse combined with a description of the spectral radiance distribution over the solar disk and its dependence on the relative distance from the centre. For the specific study, the 1-D modelled ET was used to compare its theoretical results of the LD effect on the ET, with the re- sults derived from the measurements performed during the described campaign.

\section{Total ozone column}

Surface UV-B radiation under cloud-free skies is primarily affected by atmospheric ozone and aerosols. Variation of these two parameters may partially mask the effect of the eclipse on UV-B radiation. From the DI measurements at the standard Brewer Ozone wavelengths (306.3, 310.1, 313.5, 316.8 and $320.1 \mathrm{~nm}$ ) that were conducted during the campaign with the Brewer MK III, the total ozone column has been calculated. In general, total ozone was about 30-40 DU lower on the day of the eclipse than the day before. During the course of the eclipse, one can see a gradual decrease in total ozone, followed by a symmetric increase after totality (Fig. 1). This effect has been reported by others (Zerefos et al., 2001) and it was attributed partly to the LD effect and partly to the increasing influence on DI of the diffuse radiance in the field of view of the instruments.

As mentioned in the introduction, the spectral dependence of the measured DI due to the limb darkening of the sun becomes relevant during measurements performed at an eclipse period. Due to this spectral dependent change of the ET, the wavelength pairs (ratios) that are used in the differential absorption method (Kerr, 2002) for calculating total column ozone, are also affected from the LD and result in an error on the calculated ozone. In Zerefos et al. (2001) it was suggested that the LD effect is less than $1 \%$. Here we confirm this finding by calculating a correction for total ozone based on the wavelength dependent change of ET irradiance during the eclipse derived by the model suggested in (Köpke et al., 2001). This correction is shown in the lower panel of Fig. 1, and was found to be much smaller (less than $0.01 \%$ ). Therefore the most likely reason for the apparent reduction in total ozone values during the eclipse is "contamination" of DI measurements by the diffuse radiation. Brewer DS measurements are performed with a finite field of view. Therefore there is inherently some fraction of the diffuse irradiance (aureole and Rayleigh components) entering the instrument measuring the direct irradiance (Arola and Koskela, 2004). The magnitude of this effect depends on the field of view and the wavelength but also on the air mass factor. In Fig. 1 the measurements that correspond to sun coverage by the moon of more than $70 \%$ are marked with grey triangles and should be discarded. The reduction in DI measurements at $70 \%$ eclipse coverage corresponds to an air mass factor higher than 3 , if measurements were performed under normal (non eclipse) conditions. These measurements are usually discarded in the standard Brewer total ozone measurements. For the period between 09:00 and 13:00 UT, the total ozone column was changing on the day before the eclipse from about $335 \mathrm{DU}$ down to about $325 \mathrm{DU}$, and on the day of the eclipse it was increasing from about $290 \mathrm{DU}$ to $305 \mathrm{DU}$. This large 


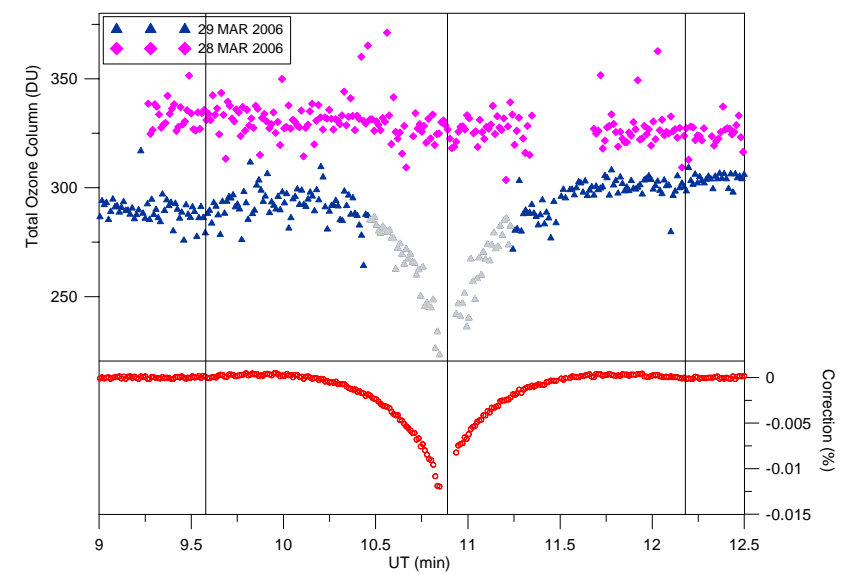

Fig. 1. Total column ozone measured by a Brewer spectroradiometer on 28 (pink) and 29 (blue) March 2006. Measurements that correspond to sun coverage by the moon of more than $70 \%$ are marked with grey triangles (red): Calculated correction in total ozone due to the limb darkening effect.

( $\sim 30$ DU) difference between the two days can be attributed to a stratospheric change of the total column ozone amount. Such large day-to-day changes are not rare for spring at the latitude that the measurements took place.

\section{Spectral measurements of solar radiation during the eclipse}

Measurements of GI and DI during the eclipse and for the previous day are shown in Fig. 2. The black vertical lines represent the start of the eclipse, totality and the end of the eclipse. Except for the reduction in both quantities due to the eclipse the main change in the irradiance is due to changes in the solar zenith angle. Local noon was within a few minutes of the start of the eclipse and all irradiance changes afterwards are a combination of the eclipse effect in addition to the reduction of the irradiance due to the decreasing solar zenith angle. The variability that is observed around 10.20 UT. and around 12:00 UT. is due to clouds passing in front of the sun. The effect is more pronounced in the DI measurements, as the GI represents the sum of the (weighted with the cosine of the solar zenith angle) DI and the diffuse irradiance components.

With the use of DI spectroradiometric data the spectral aerosol optical depth could be derived by using the BeerLambert's law and the methodology that is described in (Marenco et al., 1997). On the day before the eclipse, it was between 0.35 and 0.40 at $350 \mathrm{~nm}$, with an Angstrom exponent for the wavelength dependency of about 1 . On the day of the eclipse, similar values were measured for the aerosols.

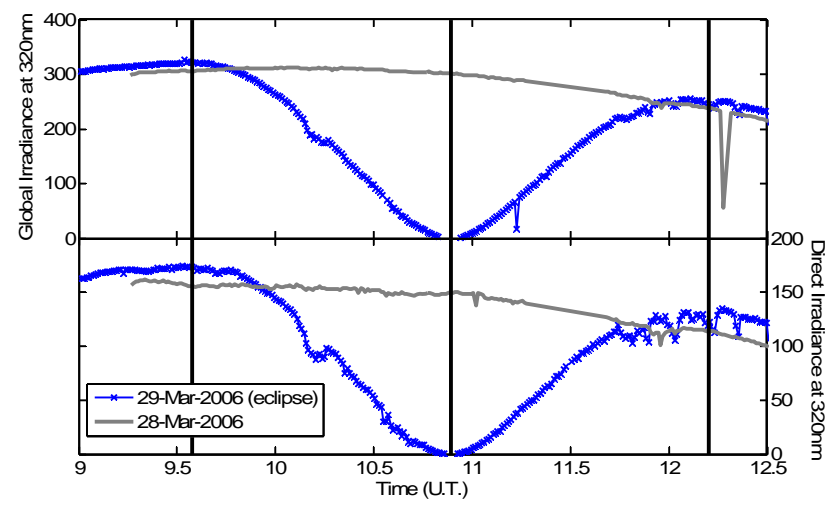

Fig. 2. Global (top) and direct (bottom) spectral irradiance at $320 \mathrm{~nm}$ measured with the Brewer spectroradiometer on the eclipse day (blue symbols) and on the previous day (grey lines).

\subsection{Global irradiance and actinic flux}

Measurements of global UV irradiance that were performed during the campaign with the NILU-UV multi-filter instrument have been used to study the spectral impact of the LD on the GI. Ratios of GI measurements at $380 \mathrm{~nm}$ to the irradiances at 312, 320 and $340 \mathrm{~nm}$ are shown in Fig. 3. First, the data were corrected for the effect of the changing solar zenith angle during the period of study by normalization with the measurements taken during the previous (cloudless) day at the same solar zenith angles. In more detail, first we have calculated the ratio $A(\lambda, \vartheta)=\left[\operatorname{GI}(\lambda, \vartheta) / \operatorname{GI}\left(\lambda, \vartheta_{0}\right)\right]$ for 28 March (cloudless conditions) (where $\lambda$ is the wavelength, $\vartheta$ the solar zenith angle and $\vartheta_{0}$ the solar zenith angle at local noon) and afterwards we have multiplied $\operatorname{GI}(\lambda, \vartheta)$ on the eclipse day with the factor $A(\lambda, \vartheta)$. These ratios were normalized again with the mean ratio over a period of 5 min just before the start of the eclipse, to bring all ratios to the same scale and allow direct comparisons for all three wavelengths. During the $5 \mathrm{~min}$ period used for the normalization the sky was free of clouds. If the attenuation of GI was spectrally independent and proportional to the geometrical sun coverage, the ratios would have been equal to unity throughout the period of the eclipse. Figure 3 shows that there is a spectral dependence which is more evident at the shorter wavelengths. The change in irradiance at 312,320 and $340 \mathrm{~nm}$ relative to the irradiance at $380 \mathrm{~nm}$, is respectively about $20 \%, 15 \%$ and $10 \%$ for $99 \%$ sun coverage. The changes of these wavelength ratios are describing how the limb darkening is affecting GI measurements at the earth's surface. This effect leads to a faster decrease of the GI at lower wavelengths than the GI at higher ones. The above given percentages indicate that for $99 \%$ sun coverage GI at $312 \mathrm{~nm}$ is decreasing $20 \%, 10 \%$ and $5 \%$ more than the GI at 380,340 and $320 \mathrm{~nm}$ respectively. For each channel, measurements of less than 10 times the dark current were eliminated from the analysis. In Fig. 3 the data that correspond to the totality and approximately $30 \mathrm{~s}$ 


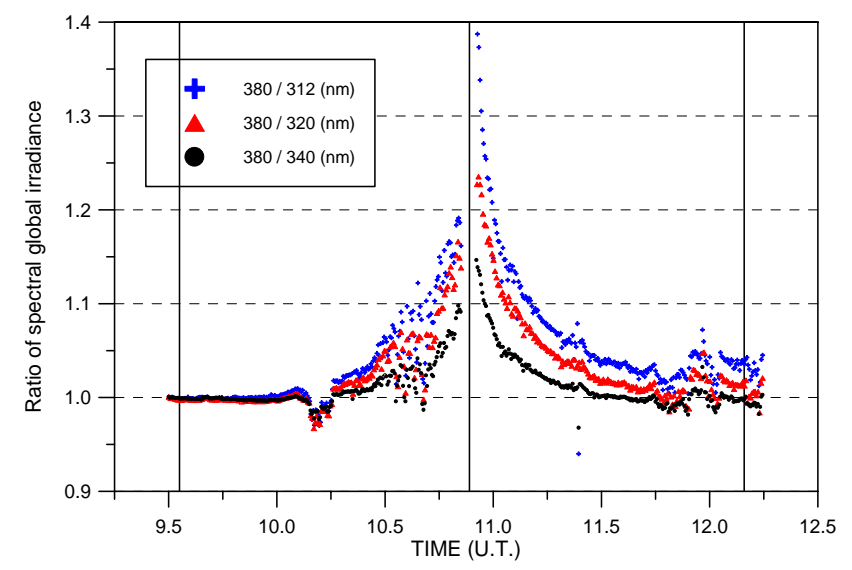

Fig. 3. Normalized and SZA corrected ratios of global irradiance at $380 \mathrm{~nm}$ relative to $312 \mathrm{~nm}$ (blue), $320 \mathrm{~nm}$ (red) and $340 \mathrm{~nm}$ (black) measured with the NILU-UV instrument during the eclipse.

before and after are not shown, because of their low signal to noise ratio.

Periods affected by clouds are visible in the dataset especially after the eclipse. In addition, the change of the total ozone column during the eclipse (see Fig. 1) results in separation of the three curves after totality (mainly after 11:00 UT). This separation occurs because the ratios for 312 and $320 \mathrm{~nm}$ are normalized with values measured at a time of lower total ozone ( 09:30 UT). GI at $340 \mathrm{~nm}$ is not affected by ozone; hence the ratio $(380 \mathrm{~nm} / 340 \mathrm{~nm})$ is more symmetric around the totality.

Measurements of AF were performed with the high frequency PDA instrument. Figure 4 shows the ratio of GI versus $\mathrm{AF}$, at 320 and $340 \mathrm{~nm}$, as measured with the two PDA instruments during the eclipse period, and the day before the eclipse. The data represent $30 \mathrm{~s}$ averages. The data during the 3 min of the totality and for a $1 \mathrm{~min}$ before and after are not shown due to the large scatter introduced by the low signal to noise ratio of the PDA's. Effects from thin cirrus clouds that have appeared occasionally during the eclipse resulted into decreases for both ratios. Generally the differences in the ratios with respect to the previous day are small (on the order of 5-8\%). As the eclipse progresses the ratio in the eclipse day becomes lower compared to the previous day, suggesting that GI is affected more by the eclipse than the $\mathrm{AF}$. The reason is the faster decrease in the direct irradiance compared to the diffuse irradiance during the eclipse which will have a more pronounced effect on GI than on AF. Due to the missing cosine weighting in the AF, the diffuse radiation far from the zenith contributes more strongly to the signal. Therefore the relative contribution of the direct component in the AF is systematically lower than in the GI (Köpke et al., 2001). RTM calculations for the specific solar zenith angles, using the measured aerosol optical depth and total ozone at Kastelorizo, showed that at $340 \mathrm{~nm}$ the contribution of the DI

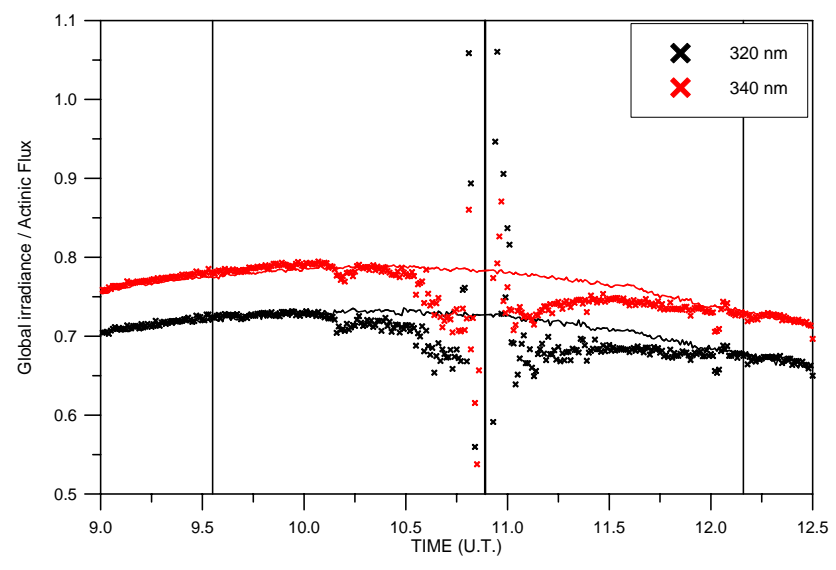

Fig. 4. Ratio of Global Irradiance/Actinic Flux at 320 (black symbols) and $340 \mathrm{~nm}$ (red symbols) as a function of time as measured with the PDA instrument. The corresponding ratios for the previous day are shown with black and red lines.

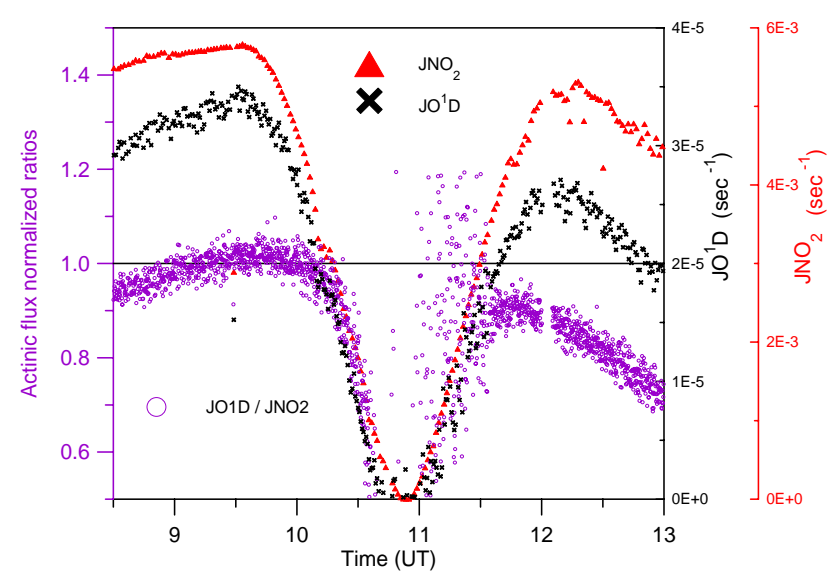

Fig. 5. Variation of $\mathrm{JO}^{1} \mathrm{D}$ (triangles) and $\mathrm{JNO}_{2}$ (crosses) photolysis rates, normalized with their values at the time of the first contact. The ratio $\mathrm{JO}^{1} \mathrm{D} / \mathrm{JNO}_{2}$ is also shown in purple circles.

is $40 \%$ for the GI and $30 \%$ for $\mathrm{AF}$. GI and $\mathrm{AF}$ values were calculated from the libRadtran model (Mayer and Kylling, 2005). The radiative transfer equation was solved with the discrete ordinates algorithm, using six streams and pseudospherical correction. The Air Force Geophysical Laboratory U.S. standard atmosphere profiles were used for ozone, temperature and air pressure. The type of landscape suggests a relatively small surface albedo, thus the spectrally constant values of 0.03 was used for the while UV region.

As it was shown, the LD effect has an impact on the GI and $\mathrm{AF}$ measured at the ground. The impact can be seen during the eclipse, by the faster decrease of the GI and AF at shorter wavelengths than the one at higher ones. As it is known, $\mathrm{JO}^{1} \mathrm{D}$ and $\mathrm{JNO}_{2}$ photolysis rates can be retrieved from the AF measurements. Furthermore, they are sensitive to the AF at different wavelength bands $\left(\mathrm{JO}^{1} \mathrm{D}\right.$ around $307 \mathrm{~nm}$ and $\mathrm{JNO}_{2}$ 

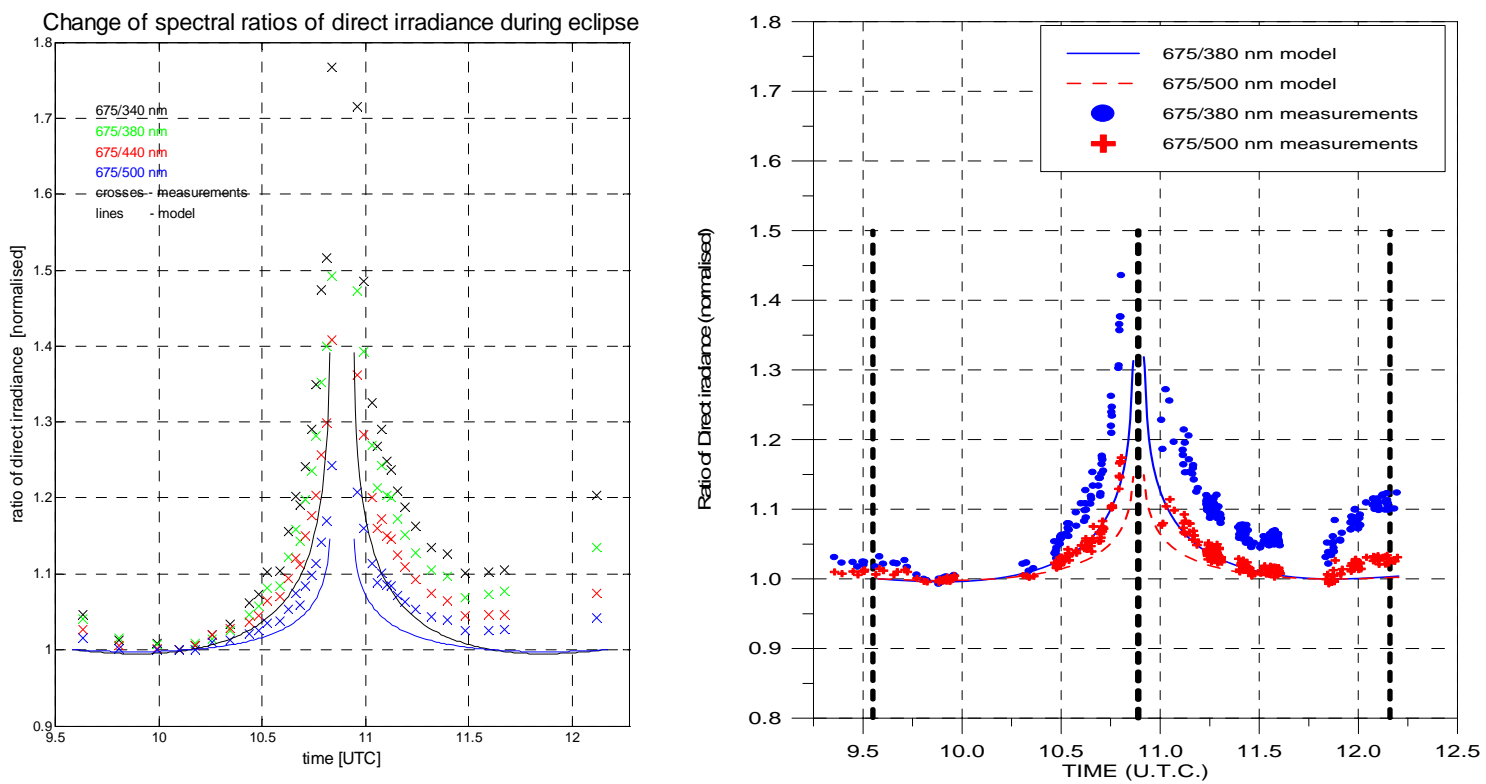

Fig. 6. Direct irradiance ratios of various wavelengths relative to $650 \mathrm{~nm}$ measured (symbols) and modeled (curves) at the time of the eclipse derived from the Microtops sun-photometer (left panel) and the CCD spectrometer (right panel).

around $380 \mathrm{~nm}$ ). Thus, the LD effect can be clearly seen also when measuring these two photolysis rates. The results in Fig. 5 show that the LD effect on the calculated photolysis rates leads to a faster decrease of the $\mathrm{JO}^{1} \mathrm{D}$ photolysis rate than the one of $\mathrm{JNO}_{2}$, as expected. Both rates and their ratio are shown in Fig. 5.

For Fig. 5 no normalization has been made to account for solar zenith angle change in order to demonstrate this solar zenith angle effect together with the LD effect on the $\mathrm{JO}^{1} \mathrm{D} / \mathrm{JNO}_{2}$ ratio.

\subsection{Direct irradiance}

The change in the spectral characteristics of the direct solar irradiance is demonstrated by the ratio of the irradiance at $675 \mathrm{~nm}$ to the irradiance at $340 \mathrm{~nm}, 380 \mathrm{~nm}, 440 \mathrm{~nm}$ and $500 \mathrm{~nm}$, which were measured by the Microtops sun photometers. The measured DI was corrected for the changing solar zenith angle according to Beer's law of extinction and as we are interested in relative changes, the above ratios were normalized to their values at around 10:00 UT. Figure 6 shows clearly the increase of these ratios during the course of the eclipse towards the totality (and the decrease after totality). A similar pattern is seen also in the ratios $675 / 340 \mathrm{~nm}$ and $675 / 500 \mathrm{~nm}$ which were derived from the STAR model calculations. Evidently, when the moon covers the sun the irradiance at shorter wavelengths is reduced more compared to longer wavelengths. However, the detail of the variation of the measured values deviates systematically from the model calculation, in the first phase of the eclipse as well as in the final phase. The measured variation is stronger than the calcu- lated one. An effect that could explain part of the differences shown in Fig. 6 is the contribution of the diffuse irradiance (entering in the field of view of the instruments) which increases as the eclipse percentage increase. This effect can not be taken into account from the model calculations presented here. The higher DI rations that can be seen after the totality compared with the ones before (left panel of Fig. 6), might indicate the effect of cirrus clouds appearing just after the total eclipse. These results for the spectral variation of the DI are very well confirmed by analyses of DI measurements with the CCD spectrometer, which show exactly the same behavior (see right panel of Fig. 6).

\section{Extraterrestrial flux and solar effective temperature calculations}

As different areas of the sun are covered during the eclipse, the radiation received at the top of the atmosphere is a combination of photons emerging from different regions of the sun (corresponding to different temperatures - Limb Darkening effect), and hence its spectrum differs from that of the black body radiation that is usually considered. Being a mixture of black body spectra of different temperatures the calculated spectrum at the top of the atmosphere, during the different phases of the eclipse, can be corresponded to a different effective temperature for each eclipse time (sun coverage percentage). In the following we estimate the ET solar spectrum which varies during the course of the eclipse and we determine the corresponding effective temperature of the 

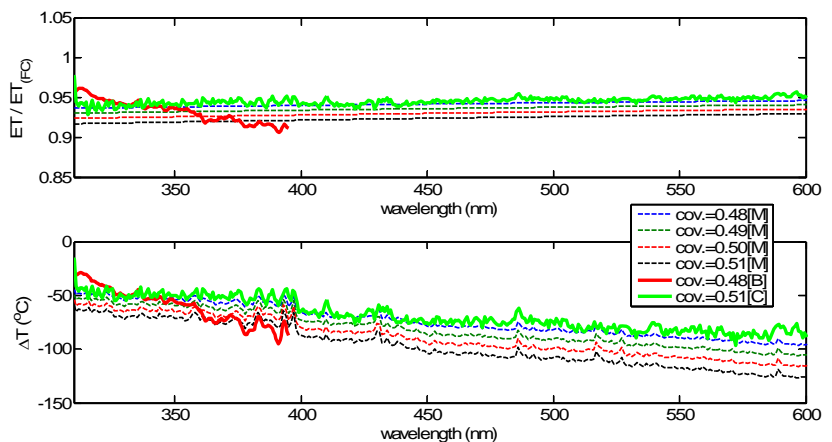

Fig. 7. (Top) ratios of calculated ET spectra at $50 \%$ sun coverage to an ET spectrum before the start of the eclipse as derived by the Bentham measurements (red line), the CCD measurements (green line) and by model calculations (dashed lines). (Bottom) Differences in effective temperature of the Sun derived from the calculated ET spectra.

partly visible sun from the equivalent to the each ET spectrum blackbody radiation using the Plank's law.

Using the Atlas 3 ET solar spectrum (Brueckner et al., 1996) and the DI measurements at the surface, the atmospheric transmission was calculated before the start of the eclipse. The ET spectrum was convolved with the slit function of each instrument and the spectral transmittance was calculated as the ratio of the measured spectral DI before the start of the eclipse (adjusted to vertical incidence) to the convolved ET solar spectrum. The derived cloudless sky transmittance, that is assumed constant during the period of the eclipse, was used to calculate new ET spectra from the measured DI at the surface during the course of the eclipse. Spectra of DI were measured by means of the scanning spectroradiometer (Bentham DTM 300), and the CCD spectrometer, while measurements at single wavelengths were provided also by the 2 handheld Microtops sun-photometers. Zerefos et al. (2001) calculated using the same methodology the pre-eclipse ET spectrum and the ET at the eclipse maximum (88\%) at Thessaloniki, Greece for the eclipse of 11 August 1999. In addition, they calculated the effective temperature derived by each wavelength in the spectral range $305-365 \mathrm{~nm}$. Here, we have extended the spectral range to $600 \mathrm{~nm}$ and we have calculated the ET spectrum change and the corresponding effective temperature for all measurement points (times) during the eclipse. The analysis shown hereafter is restricted to the first part of the eclipse only, as after the totality more cirrus clouds appeared on the sky causing changes in the atmospheric transmission.

In the upper plot of Fig. 7, the spectral ratio of the ET flux as calculated for $50 \%$ sun coverage to the ET flux before the start of the eclipse is shown. Red and green lines represent measurements with the Bentham spectroradiometer (for $48 \%$ sun coverage) and the CCD spectrometer (for $51 \%$ sun coverage) respectively. The dashed lines represent

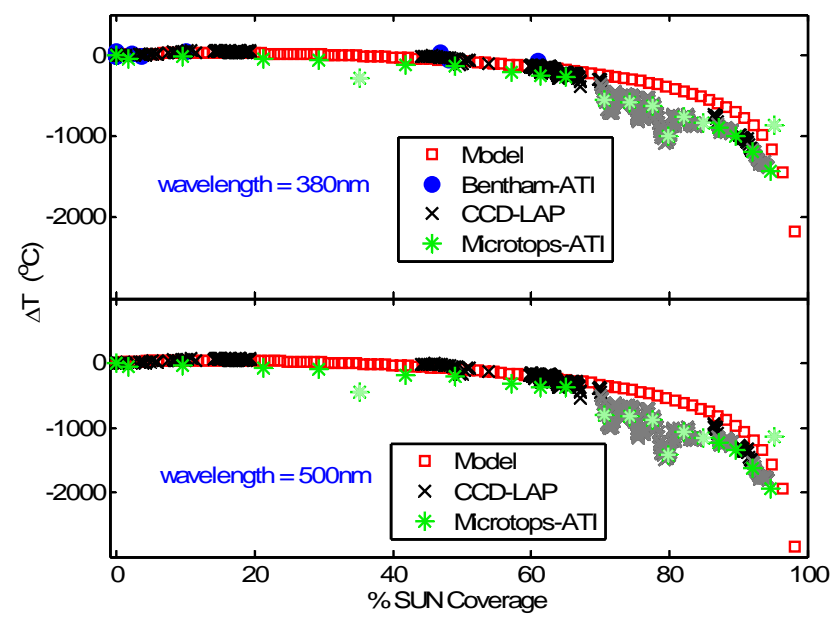

Fig. 8. Solar effective temperature difference from the temperature at the start of the eclipse as a function of sun coverage derived from irradiance measurements at $380 \mathrm{~nm}$ (top) and $500 \mathrm{~nm}$ (bottom) from three instruments and STAR model data. Grey symbols represent cloud infected measurements.

the STAR model calculations for different fractions of sun coverage from $48 \%$ to $51 \%$, in steps of $1 \%$. The lower plot of Fig. 7 shows the difference of the sun effective temperature calculated at the specific time of the eclipse from the temperature calculated before the start of the eclipse. It has to been mentioned that we compared the ET at no eclipse conditions with the measured (from DI) ET's at certain times (sun coverage percentages) during the eclipse. The latter was corrected (normalized) dividing it with the percentage of geometrical sun coverage at each point. For example the 50\% eclipse ET (shown in the upper plot of Fig. 7) was first calculated from the DI measurements, then divided with a factor of 0.5 and finally compared with the non eclipse ET. The difference of the two is due to the limb darkening effect. If this effect was not present, all ET ratios would be equal to one and all temperature differences would be equal to zero for all wavelengths. We have calculated these ratios and temperature differences for various points (times) during the eclipse (shown in Fig. 8) while Zerefos et al. (2001) have calculated the same feature only for the maximum ( $88 \%$ sun coverage) of the eclipse. This has been investigated in order to show the spectral deviations, due to the LD effect, of the calculated ET's from the ones calculated with the use of the Planck's law. In this case, the LD effect creates a temperature difference that varies with wavelength. It should be mentioned that the CCD measures a spectrum in less than $20 \mathrm{~s}$ while the Bentham spectroradiometer measurement lasts for about $3 \mathrm{~min}$, which in conjunction to the changing conditions due to the eclipse leads to differences in the measurements of the two instruments. This can explain part of the observed differences in the calculated ET spectra derived from the direct irradiance measurements by the two instruments, as shown in the upper panel of Fig. 7. 
An interesting effect can be seen when we calculated the same ET flux ratios and temperature differences for the first minutes of the eclipse. For $10 \%$ sun coverage both instruments and the model calculations show an increase in the calculated ET flux of up to $5 \%$ and also a temperature increase (up to $40^{\circ}$ ). This happens because in the beginning of the eclipse only the outer part of the solar disk is covered. The outer part has a lower temperature so the mean temperature of the sun at this moment is dominated by the temperature of the central part of the solar disk. This leads to the calculation of a higher ET than the one calculated before the start of the eclipse.

The deviation of the solar effective temperature derived indirectly from the measurements from the one calculated assuming the black body emission for two wavelengths $(380 \mathrm{~nm}$ and $500 \mathrm{~nm}$ ) is shown in Fig. 8 for the different phases of the eclipse. Grey symbols represent DI measurements with the CCD spectrometer that were likely affected by cirrus clouds. As a result of this change in atmospheric transmission the calculated ET flux is lower than the one expected under cloudless skies, and thus the calculated effective temperatures are lower than those predicted by the model. The results from all instruments show similar behavior. Larger deviations are observed for longer wavelengths and an acceleration of the rate of this decrease is observed in the last minutes before totality. In general, modeled ET's and ET's inferred from measurements, during times not disturbed by clouds, show mostly good agreement.

\section{Conclusions}

Spectral measurements of the solar radiation were performed at Kastelorizo, Greece during a total solar eclipse. The results were compared with changes in the extraterrestrial solar flux predicted according to the technique explained in Köpke et al. (2001). The model results allow the computation of a correction for the total ozone measurements due to the limb darkening effect during the eclipse. This correction is far too small to explain the large decrease in total ozone column derived from the standard Brewer measurements. It is suggested that this decrease in total ozone is an artifact due to the increasing contribution of diffuse radiation against the decreasing direct irradiance (that is used for the total ozone column retrieval) caused by the eclipse.

Global irradiance, direct irradiance and actinic flux measurements showed that all quantities are spectrally affected by the limb darkening during the eclipse. The effect leads to wavelength dependent changes in the measured solar spectra showing a much more pronounced decrease in the radiation at the lower wavelengths as the percentage of the sun coverage is increased for all the above quantities.

The comparison of model results and measurements showed that previous 1-D model calculations underestimate this spectral limb darkening effect especially close to the totality of the solar eclipse. 3-D model calculations performed by Emde and Mayer (2007) reported that 3-D calculations show that the radiation decreases much more for shorter wavelengths than the 1-D model would suggest due to limb darkening. They also quantified the uncertainty of the 1-D approximation at different times of the eclipse (eclipse phases). This result was confirmed by measurements from two different instruments presented in this study.

Calculations of the ET solar spectrum and the effective sun's temperature as derived from direct irradiance measurements at the surface, showed an artificial change in both quantities. The limb darkening effect induces changes in the ET spectrum measured with the help of remote sensing techniques. The derived ET spectrum is a mixture of black body radiation spectra originating from parts of the solar disk with different temperatures. Thus, fitting a Plank function on these data, in order to derive the corresponding black body temperature of the Sun, leads to false estimates.

Acknowledgements. The authors would like to acknowledge the anonymous referees for their work on the substantial improvement of the manuscript.

Edited by: C. Zerefos

\section{References}

Arola, A. and Koskela, T.: On the sources of bias in aerosol optical depth retrieval in the UV range, J. Geophys. Res., 109, D08209, doi:10.1029/2003JD004375, 2004.

Bais, A. F., Gardiner, B. G., Slaper, H., Blumthaler, M., Bernhard, G., McKenzie, R., Webb, A. R., Seckmeyer, G., Kjeldstad, B., Koskela, T., Kirsch, P. J., Gröbner, J., Kerr, J. B., Kazadzis, S., Leszcznski, K., Wardle, D., Josefsson, W., Brogniez, C., Gillotay, D., Reinen, H., Weihs, P., Svenoe, T., Eriksen, P., Kuik, F., and Redondas A.: SUSPEN intercomparison of ultraviolet spectroradiometers, J. Geophys. Res.-Atmos., 106(D12), 12 509-12 525, 2001.

Beletsky, A. B., Mikhalev, A. V., and Chernigovskaya, M. A.: Spectral measurements of the solar nearground UV radiation during the solar eclipse on March 9, 1997, Atmos. Oceanic Opt., 11, 301-306, 1998.

Bojkov, R. D.: The ozone variations during the solar eclipse of May 20 1966, Tellus, 20, 417-421, 1968.

Brueckner, L., Floyd, E., Lund, P. A., Prinz, D. K., and VanHoosier, M. E.: Solar Ultraviolet Spectral Irradiance Observations for the UARS/SUSIM Experiment, G.E., Metrologia, 32, 661-665, 1996.

Chakrabarty, D. K., Shah, N. C., and Pandya, K. V.: Fluctuation in ozone column over Ahmedabad during the solar eclipse of 24 October 1995, Geophys. Res. Lett., 24(23), 3001-3004, 1997.

Emde, C. and Mayer, B.: Simulation of solar radiation during a total solar eclipse: A challenge for radiative transfer, Atmos. Chem. Phys., 7, 2259-2270, 2007,

http://www.atmos-chem-phys.net/7/2259/2007/.

Fernandez, W., Castro, V., Wright, J., Hidalgo, H., and Saenz, A.: Changes in solar irradiance and atmospheric turbidity in Costa 
Rica during the total solar eclipse of July 11, 1991, Earth Moon Planets, 63(2), 119-132, 1993.

Greve, A. and Neckel, H.: On the consistency of solar limb darkening observations at UV wavelengths (2000-3300 A), Astronomy and Astrophysics Supplement Series, 120(1), 35-39, 1996.

Jerlov, N., Olsson, H., and Schuepp, W.: Measurements of solar radiation at Loevanger in Sweden during the total eclipse 1945, Tellus, 6, 44-45, 1954.

Kerr, J. B.: New methodology for deriving total ozone and other atmospheric variables from Brewer spectrophotometer direct Sun spectra, J. Geophys. Res., 107(D23), 4731, doi:10.1029/2001JD001227, 2002.

Köpke, P., Reuder, J., and Schween, J.: Spectral variation of the solar radiation during an eclipse, Meteorol. Z., 10(3), 179-186, 2001.

Kylling, A., Webb, A. R., Kift, R., et al.: Spectral actinic flux in the lower troposphere: measurement and 1-D simulations for cloudless, broken cloud and overcast situations, Atmos. Chem. Phys., 5, 1975-1997, 2005, http://www.atmos-chem-phys.net/5/1975/2005/.

Marenco, F., Santacesaria, V., Bais, A. F., Balis, D., Di Sarra, A., Papayannis, A., and Zerefos, C. S.: Optical properties of tropospheric aerosols determined by lidar and spectrophotometric measurements (PAUR campaign), Appl. Opt., 36, 6875-6886, 1997.

Mayer, B. and Kylling, A.: Technical note: The libRadtransoftware package for radiative transfer calculations - description and examples of use, Atmos. Chem. Phys., 5, 1855-1877, 2005, http://www.atmos-chem-phys.net/5/1855/2005/.

Mikhalev, A. V., Chernigovskaya, M. A., Beletsky, A. B., Kazimirovsky, E. S., and Pirog, O. M.: Variations of the groundmeasured solar ultraviolet radiation during the solar eclipse on March 9, 1997, Adv. Space Res., 24(5), 611-619, 1999.

Nakajima, T. and Tanaka, M.: Algorithms for radiative intensity calculations in moderately thick atmospheres using a truncation approximation, J. Quant. Spectrosc. Radiat. Trans., 40, 51-69, 1988.
Scheffler, H. and Elsässer, H.: Physik der Sterne und der Sonne, 535 pp., Bibliographisches Institut Zürich, Zurich, Switzerland, 1974.

Shaw, G. E.: Sky radiance during a solar eclipse: a theoretical model, Appl. Opt., 17, 272-278, 1978.

Sharp, W. E., Silverman, S. M., and Lloyd, J. W. F.: Summary of sky brightness measurements during eclipses of the sun, Appl. Opt., 10, 1207-1210, 1971.

Schwander, H., Kaifel, A., Ruggaber, A., and Koepke, P.: Spectral radiative-transfermodeling with minimized computation time by use of a neural-network technique, Appl. Opt., 40, 331-335, 2001.

Stranz, D.: Ozone measurements during solar eclipse, Tellus, 13, 276-279, 1961.

Svensson, B.: Observations on the amount of ozone by Dobson spectrophotometer during the solar eclipse of June 30, 1954, Arkives Geofysikae, 2, 573-594, 1958.

Waldmeier, M.: Ergebnisse und Probleme der Sonnenforschung, Probleme der kosmischen Physik, 264 pp., Akad Verlagsges Becker \& Erler, Leipzig, Germany, 1941.

Webb, A. R., Bais, A. F., Blumthaler, M., Gobbi, G. P., Kylling, A., Schmitt, R., Thiel, S., Barnaba, F., Danielsen, T., Junkermann, W., Kazantzidis, A., Kelly, P., Kift, R., Liberti, G. L., Misslbeck, M., Schallhart, B., Schreder, J., and Topaloglou, C.: Measuring spectral actinic flux and irradiance: Experimental results from the Actinic Flux Determination from Measurements of Irradiance (ADMIRA) project, J. Atmos. Ocean. Tech., 19(7), 1049-1062, 2002.

Zerefos, C. S., Balis, D. S., Zanis, P., Meleti, C., Bais, A. F., Tourpali, K., Melas, D., Ziomas, I., Galani, E., Kourtidis, K., Papayannis, A., and Gogosheva, Z.: Changes in surface UV solar irradiance and ozone over the Balkans during the eclipse of August 11, 1999, Adv. Space Res., 27(12), 1955-1963, 2001. 